\title{
Effects of camptothecin derivatives and topoisomerase dual inhibitors on Trypanosoma cruzi growth and ultrastructure
}

\author{
Otto Kischlat Lacombe ${ }^{1 \dagger}$, Aline Araujo Zuma ${ }^{1+}$, Camila Cristina da Silva ${ }^{1}$, Wanderley de Souza $a^{1,2,3}$
} and Maria Cristina M Motta ${ }^{*}$

\begin{abstract}
Background: Trypanosoma cruzi is the etiological agent of Chagas' disease that is an endemic disease in Latin America and affects about 8 million people. This parasite belongs to the Trypanosomatidae family which contains a single mitochondrion with an enlarged region, named kinetoplast that harbors the mitochondrial DNA (kDNA). The kinetoplast and the nucleus present a great variety of essential enzymes involved in DNA replication and topology, including DNA topoisomerases. Such enzymes are considered to be promising molecular targets for cancer treatment and for antiparasitic chemotherapy. In this work, the proliferation and ultrastructure of T. cruzi epimastigotes were evaluated after treatment with eukaryotic topoisomerase I inhibitors, such as topotecan and irinotecan, as well as with dual inhibitors (compounds that block eukaryotic topoisomerase I and topoisomerase II activities), such as baicalein, luteolin and evodiamine. Previous studies have shown that such inhibitors were able to block the growth of tumor cells, however most of them have never been tested on trypanosomatids.
\end{abstract}

Results: Considering the effects of topoisomerase I inhibitors, our results showed that topotecan decreased cell proliferation and caused unpacking of nuclear heterochromatin, however none of these alterations were observed after treatment with irinotecan. The dual inhibitors baicalein and evodiamine decreased cell growth; however the nuclear and kinetoplast ultrastructures were not affected.

Conclusions: Taken together, our data showed that camptothecin is more efficient than its derivatives in decreasing T. cruzi proliferation. Furthermore, we conclude that drugs pertaining to a certain class of topoisomerase inhibitors may present different efficiencies as chemotherapeutical agents.

Keywords: Cell proliferation, Kinetoplast, Nucleus, Topoisomerase inhibitors, Trypanosomatid protozoa, Ultrastructure

\section{Introduction}

The Trypanosomatidae family comprises protozoa of medical and veterinary importance. This group includes species that are the etiological agents of numerous human diseases, such as Chagas' disease (caused by Trypanosoma cruzi), African sleeping sickness (caused by Trypanosoma brucei), and leishmaniasis (caused by Leishmania spp). Chagas' disease was discovered in 1909 and nowadays

\footnotetext{
* Correspondence: motta@biof.ufrj.br

${ }^{\dagger}$ Equal contributors

'Laboratório de Ultraestrutura Celular Hertha Meyer, Instituto de Biofísica Carlos Chagas Filho, Universidade Federal do Rio de Janeiro, 21491-590 Rio de Janeiro, RJ, Brazil

Full list of author information is available at the end of the article
}

affects about 8 million people in Latin America and new cases are being reported in non-endemic areas due to emigrations [1].

T. cruzi is a flagellated protozoan and like other trypanosomatids presents a single mitochondrion with an enlarged region, termed kinetoplast, which contains the mitochondrial DNA (kDNA). T. cruzi also has a single spherical nucleus presenting a condensed heterochromatin next to the nuclear envelope and around the nucleolus [2-6]. Since the nucleus and the kinetoplast are cellular compartments that contain DNA, their structural organization depends on enzymes such as topoisomerases, that play a key role during replication, transcription, recombination and repair [7-9]. 
DNA topoisomerases are classified into type I and type II. Type I attaches to DNA and breaks one strand of the double helix that can rotate around its own axis to revert supercoiling. On the other hand, type II binds to a DNA double strand and makes a gate allowing a second DNA double helix pass [10].

Several topoisomerase inhibitors have been developed based on different types of these enzymes that have been considered as potent targets in chemotherapeutic studies, especially with tumor cells. Topo I inhibitors, such as camptothecin, form a ternary complex, since they can trap the enzyme and DNA together [11-14]. Topo II inhibitors, such as mitoxantrone and etoposide, bind to the enzyme preventing the re-ligation of the DNA double strand. Furthermore, some inhibitors share characteristics of the two groups described above and target both topo I and topo II, thereby being called dual inhibitors [15].

Many topoisomerase inhibitors are natural products extracted from plants, such as camptothecin, isolated from Camptotheca accuminata, and several alkaloids, such as evodiamine, isolated from Evodia rutaecarpa [16]. Topotecan and irinotecan are camptothecin derivatives that have been used for ovarian and colorectal cancer treatments, respectively. These inhibitors target topo I and bind to DNA, forming a cleavable complex. The collision between this ternary complex and a replication fork generates DNA double-strand breaks, which may be related to the S-phase cytotoxicity, the G2/M cell cycle arrest and DNA damage that activates repair proteins [14].

Baicalein, luteolin and evodiamine are topoisomerase dual inhibitors. Baicalein is an alkaloid isolated from Scutellaria baicalensis used in the treatment of hypertension, atherosclerosis, dysentery and inflammatory diseases [16]. Luteolin is a flavonoid, a group of natural compounds with therapeutic properties that causes apoptosis in promastigote forms of $L$. donovani [17-19]. Evodiamine is an alkaloid extracted from Evodia rutecarpa used as an anticancer, anti-inflammatory and antiobesity agent [20]. This compound was initially classified as a topo I inhibitor, but then it was proposed that evodiamine could also bind to topoisomerase II [21].

In the present work, we evaluated the effects of the eukaryotic topoisomerase I inhibitors, topotecan and irinotecan, and the eukaryotic dual inhibitors baicalein, luteolin and evodiamine on the epimastigote forms of T. cruzi, considering its proliferation and ultrastructural organization.

\section{Materials and methods Protozoa culture}

T. cruzi epimastigote forms were grown at $28^{\circ} \mathrm{C}$ for $24 \mathrm{~h}$ in liver infusion tryptose (LIT) medium [22] supplemented with $10 \%$ fetal calf serum.

\section{Drug treatment}

Topotecan, irinotecan, baicalein, luteolin and evodiamine were purchased from Sigma Aldrich and diluted in dimethyl sulfoxide (DMSO) at $5 \mathrm{mM}$ and $30 \mathrm{mM}$. The drug was added to the culture medium after $24 \mathrm{~h}$ of initial growth, which corresponds to the exponential phase. Drug concentrations were used as follows: 1, 5, 10, 50, 100, 200 and $300 \mu \mathrm{M}$. Every $24 \mathrm{~h}$ cells were collected and counted in a Neubauer chamber during the $96 \mathrm{~h}$ of cultivation. Paired t-tests were applied to the results using 95\% confidence interval (GraphPad Prism version 5.00 for windows; GraphPad Software Inc., San Diego, CA).

Cell viability was performed using the MTS/PMS colorimetric method, which is based on dehydrogenase activity and the conversion of MTS into formazan, that indicates the number of metabolically active cells [23]. Parasites were incubated with MTS/PMS solution for $4 \mathrm{~h}$ and formaldehyde $0.4 \%$ was used as negative control. The percentage of viable protozoa was obtained through a spectrofluorimeter (Molecular Devices Microplate Reader (SpectraMax M2/M2 ${ }^{\mathrm{e}}$, Molecular Devices) using a $490 \mathrm{~nm}$ wavelength. MTS/PMS is a colorimetric assay, based on dehydrogenase activity and the conversion of MTS into formazan, that indicates the number of metabolically active cells.

\section{Transmission electron microscopy}

Protozoa were fixed in $2.5 \%$ glutaraldehyde diluted in $0.1 \mathrm{M}$ cacodylate buffer ( $\mathrm{pH}$ 7.2) for $1 \mathrm{~h}$ at room temperature and were washed in the same buffer. Cells were post-fixed for $1 \mathrm{~h}$ in $0.1 \mathrm{M}$ cacodylate buffer containing $1 \% \mathrm{OsO}_{4}$ and $0.8 \%$ potassium ferricyanide. Protozoa were washed in the same buffer and were dehydrated in a graded series of acetone and embedded in Epon (Electron Microscopy Sciences, Hatfield, PA). Ultrathin sections were stained with uranyl acetate and lead citrate and were observed using a Zeiss 900 transmission electron microscope (Zeiss, Oberkochen, Germany).

\section{Results}

Regarding topoisomerase I inhibitors, cell proliferation was not significantly affected by topotecan after treatment with $50 \mu \mathrm{M}$ for $72 \mathrm{~h}$, while up to $300 \mu \mathrm{M}$ reduced cell proliferation by approximately 3 fold in relation to the control cells was observed (Figure 1a), resulting in IC50 value of $110 \mu \mathrm{M}$. It is interesting to point out that induction of cell growth inhibition was noted after $48 \mathrm{~h}$ of treatment. Furthermore, this compound induced cell viability decay in a dose dependent manner, which corresponds to $20 \%$ after protozoa cultivation in medium containing 200 or $300 \mu \mathrm{M}$ topotecan for $72 \mathrm{~h}$ (Figure $1 \mathrm{~b}$ ). On the other hand, irinotecan did not promote growth impairment with any of the concentrations tested (Figure 1c). Also cell viability was not affected (data not shown for Additional file 1). 

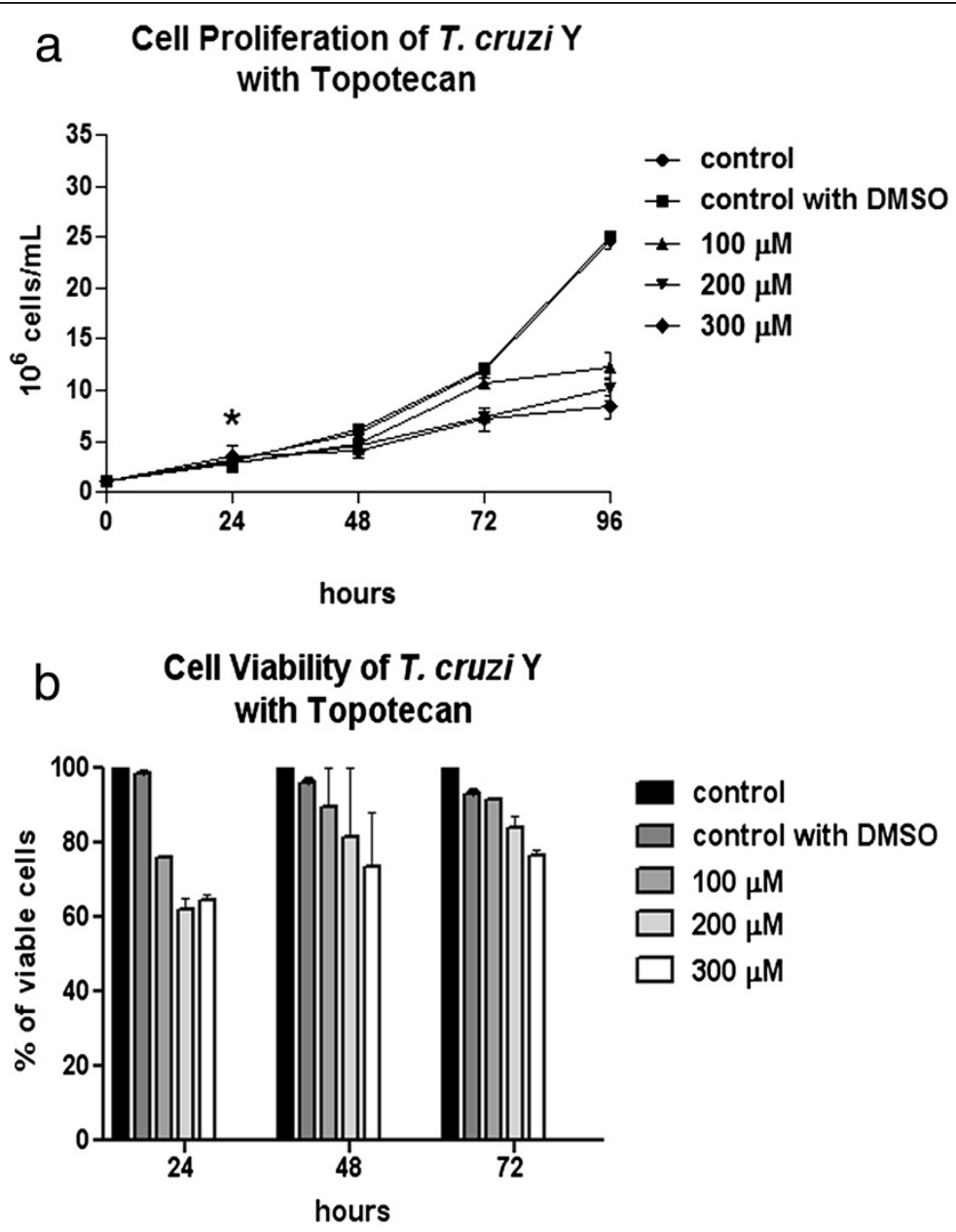

\section{Cell Proliferation of $T$. cruzi Y
with Irinotecan}

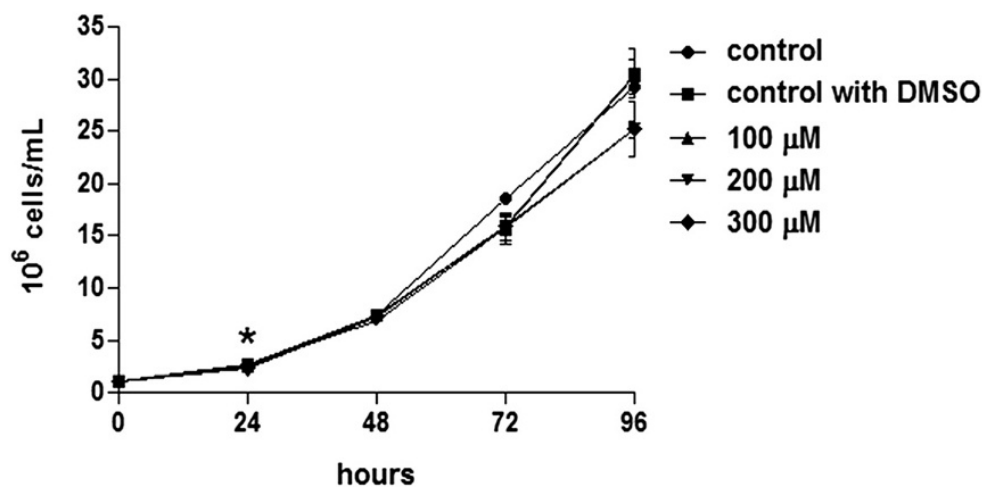

Figure 1 The effects of topoisomerase I inhibitors on $T$. cruzi epimastigotes after $\mathbf{7 2} \mathbf{h}$ of treatment. (a) Topotecan affected cell proliferation especially after using higher drug concentrations. (b) Cell viability of $T$. cruzi treated with topotecan was reduced in a dose-dependent way after treatment for 48 h. (c) Irinotecan did not cause a significant growth inhibition even after treatment for 72 h. The asterisks indicate drug addition to the culture medium. Data are the average of three independent experiments. DMSO, dimethyl sulfoxide.

Transmission electron microscopy was used to study the ultrastructural modifications caused by these inhibitors. After treatment with lower concentrations, $T$. cruzi ultrastructure was similar to control cells (Figure 2a). However protozoa cultivation with $300 \mu \mathrm{M}$ topotecan for $72 \mathrm{~h}$ led to an unpacking of the nuclear heterochromatin around the 


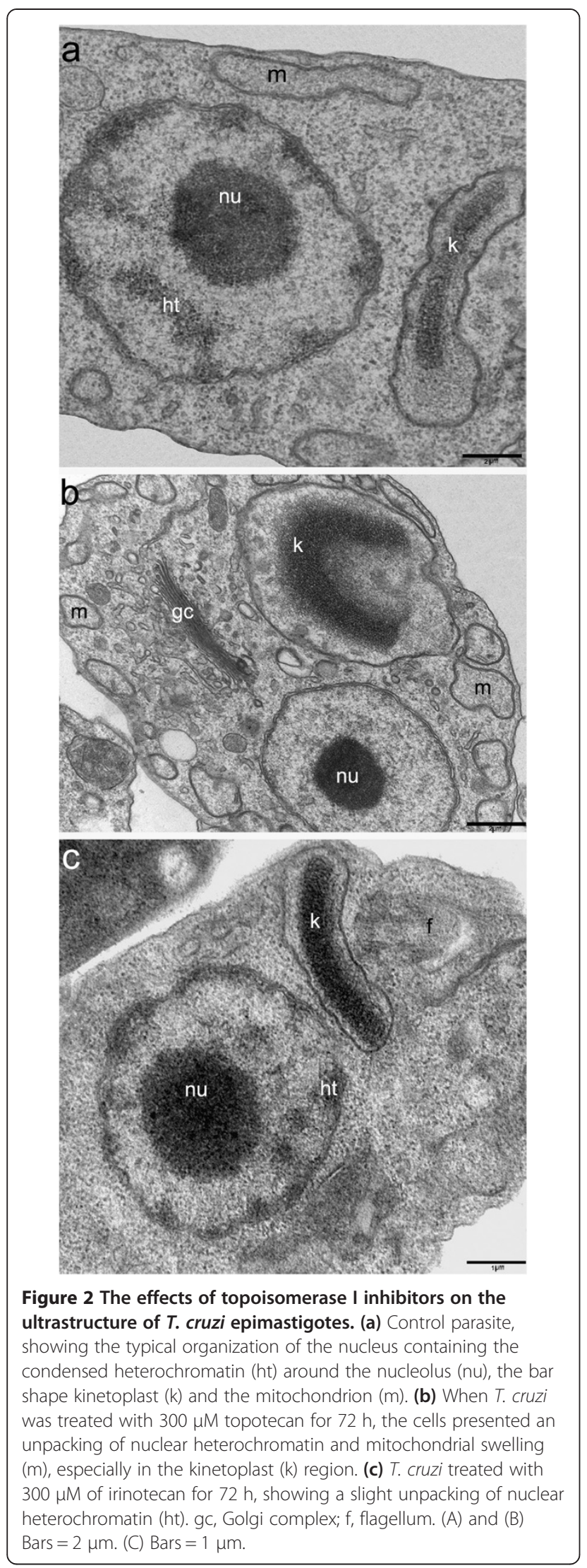

nucleolus and juxtaposed to the nuclear envelope. Mitochondrial swelling was also observed, especially in the kinetoplast region; however the kDNA arrangement was not affected (Figure 2b). Like the lack of effect in cell proliferation, irinotecan did not cause any significant changes in the cell ultrastructure. The main effect observed was a slight unpacking of heterochromatin (Figure 2c) and mitochondrial swelling, but with less intensity when compared to protozoa treated with topotecan.

The dual inhibitor baicalein affected cell proliferation in a dose-dependent manner (Figure 3a), resulting in an $\mathrm{IC}_{50}$ of $62.83 \mu \mathrm{M}$ after $72 \mathrm{~h}$. Treatment with $300 \mu \mathrm{M}$ baicalein for $24 \mathrm{~h}$ promoted a decrease of approximately 3 fold in parasite number and this difference increased to about 24 fold after $72 \mathrm{~h}$ (Figure 3a). Similar results were observed in cells treated with $200 \mu \mathrm{M}$ baicalein, whereas the concentration of $100 \mu \mathrm{M}$ reduced protozoa proliferation, but did not promote cell growth arrest. Luteolin was not able to inhibit protozoa growth significantly even after treatment with $300 \mu \mathrm{M}$ for $72 \mathrm{~h}$ (Figure $3 \mathrm{~b}$ ). Evodiamine only promoted an expressive decay in cell proliferation after using concentrations equal or superior to $100 \mu \mathrm{M}$, when it was possible to observe a dose-dependent decrease in growth (Figure 3c), which is lower when compared to that caused by baicalein (Figure 3a). Regarding cell viability, after comparing the effect of these topoisomerase dual inhibitors, it is possible to conclude that baicalein was the most potent drug. This compound reduced the percentage of viable protozoa to approximately $30 \%$ after treatment with $200 \mu \mathrm{M}$ for $24 \mathrm{~h}$ and this value was inferior to $20 \%$ after using $200 \mu \mathrm{M}$ for $72 \mathrm{~h}$ (Figure $4 \mathrm{a}$ ). The effect of evodiamine on cell viability was less intense than that observed for baicalein, but interestingly it was more pronounced in cells treated for $24 \mathrm{~h}$ (Figure $4 \mathrm{~b}$ ). Luteolin did not interfere in cell viability (data not shown for Additional file 2).

In terms of T. cruzi ultrastructure, the parasites treated with dual inhibitors did not present alterations when compared to the control cells. These compounds did not lead to modifications in the kDNA topology or in the heterochromatin organization, as was observed after treatment with topo I inhibitors (data not shown for Additional file 3).

Table 1 summarizes data obtained in this work showing the $\mathrm{IC}_{50}$ values and the main ultrastructural alterations caused by topoisomerase I and dual inhibitors to the T. cruzi epimastigotes.

\section{Discussion}

In the present work, the effects of different topoisomerase inhibitors were evaluated considering $T$. cruzi proliferation and ultrastructure. Irinotecan and topotecan are derivatives of camptothecin, thus they act by binding to DNA and to topoisomerase I by forming a ternary complex, referred to as a cleavable complex. These compounds 


\section{a Cell proliferation of $T$. cruzi Y with Baicalein}

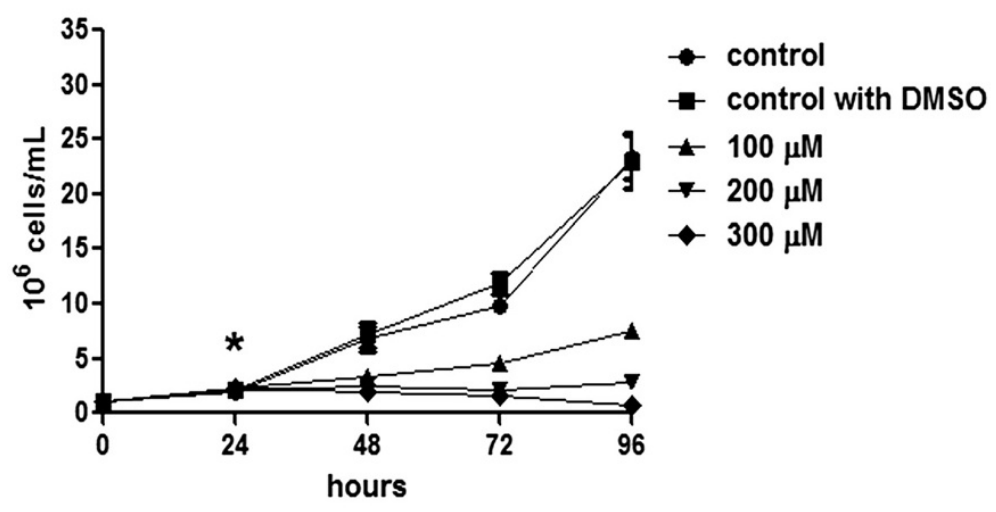

\section{b Cell Proliferation of T. cruzi Y with Luteolin}

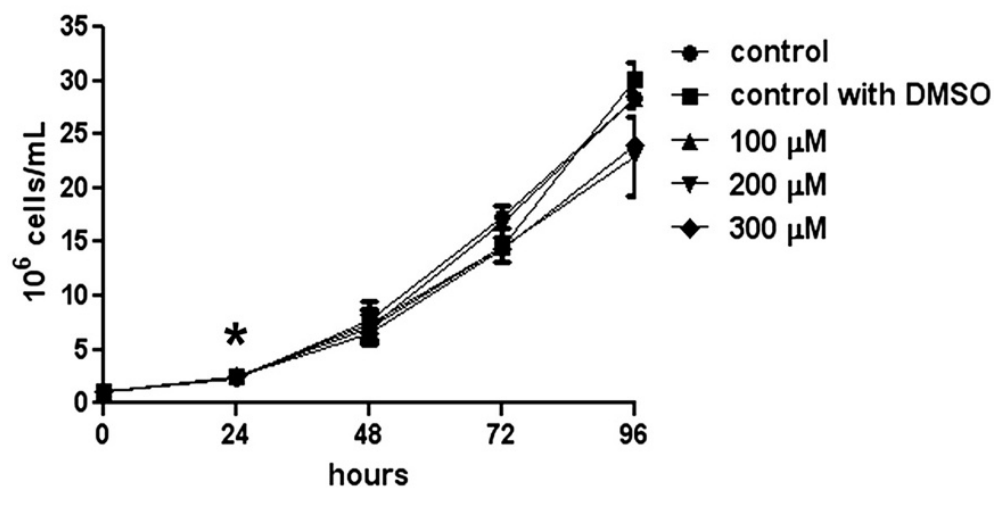

C Cell proliferation of T. cruzi Y

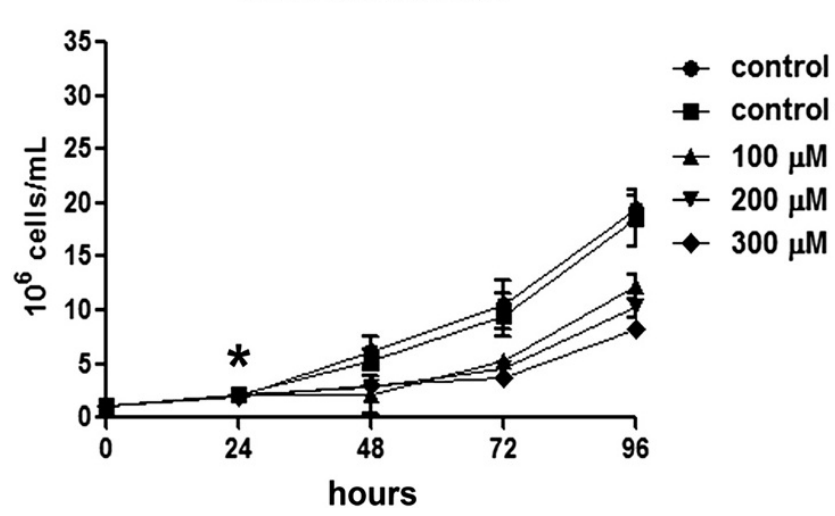

Figure $\mathbf{3}$ The effects of dual inhibitors on $\mathbf{T}$. cruzi epimastigotes after $\mathbf{7 2} \mathbf{h}$ of treatment. (a) Baicalein considerably reduced protozoa proliferation in a dose-dependent manner. (b) Luteolin did not promote growth impairment. (c) Evodiamine caused a reduction of parasite proliferation, but values were very similar with different drug concentrations. The asterisks indicate drug addition to the culture medium. Data are the average of three independent experiments. DMSO, dimethyl sulfoxide.

interfere with the re-join of the double-strand break, leading to cell cycle blockade, activation of DNA repair and apoptosis [24].
Here, we observed that topotecan promoted a moderate effect on cell proliferation, whereas irinotecan did not affect protozoa growth. These results revealed that such inhibitors 

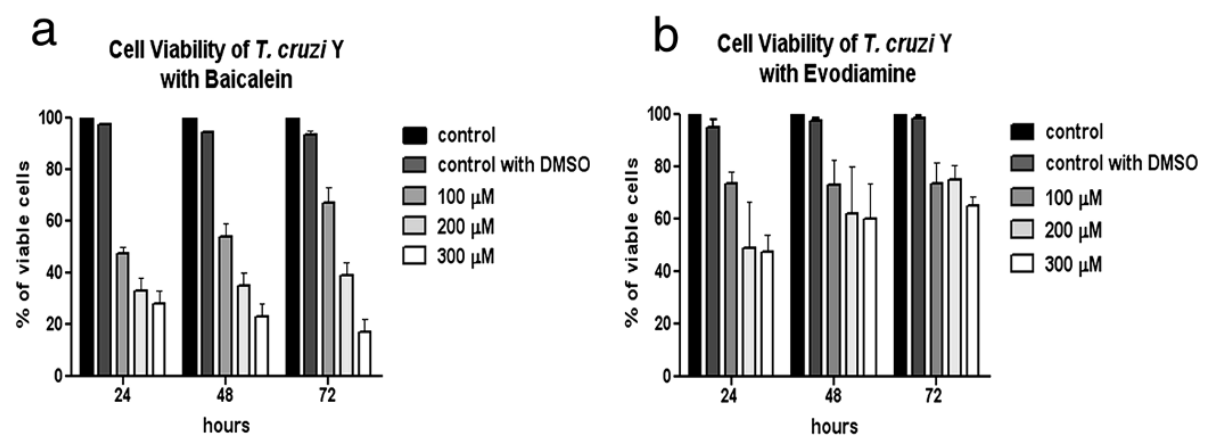

Figure 4 The effects of baicalein and evodiamine on $T$. cruzi viability after $72 \mathrm{~h}$ of treatment. (a) The number of viable cells was strongly reduced after treatment with baicalein. (b) Evodiamine caused a lower reduction of cell viability when compared to baicalein. Data are the average of three independent experiments. DMSO, dimethyl sulfoxide.

were not efficient in impairing T. cruzi growth when compared to camptothecin, the precursor compound, which presented $\mathrm{IC}_{50}$ values of $2.08 \mu \mathrm{M}$. The typical ultrastructural alterations, such as the unpacking of nuclear heterochromatin, promoted by topoisomerase I inhibitors were observed in cells treated with topotecan; however such modifications were only noticed after using high drug concentrations [25].

As described previously, topotecan and irinotecan were able to inhibit tumor cell proliferation, and were more effective and less toxic than camptothecin [26,27]. Such effects have also been reported on T. brucei and on Leishmania infantum promastigotes. In both these trypanosomatid species topotecan presented more efficacy than irinotecan, especially in $T$. brucei. The $\mathrm{IC}_{50}$ values correspond to $1.23 \mu \mathrm{M}$ for topotecan and $21.5 \mu \mathrm{M}$ for irinotecan on T. brucei, whereas values are equivalent to $10.86 \mu \mathrm{M}$ for topotecan and superior to $200 \mu \mathrm{M}$ for irinotecan on L. infantum $[28,29]$. A previous work also showed that camptothecin was cytotoxic to T. brucei and L. donovani, with $\mathrm{IC}_{50}$ values ranging from 1 to $3 \mu \mathrm{M}$ [30].

Baicalein was the most effective compound against $T$. cruzi proliferation and viability considering all the inhibitors evaluated in this study. The treatment of Leishmania promastigotes with concentrations inferior to $15 \mu \mathrm{M}$ of baicalein for $24 \mathrm{~h}$ was previously reported to reduce parasite growth up to $89 \%$ [31]. Furthermore, published data demonstrated that this drug inhibited tumor cell growth in vitro and in vivo and presented low toxicity $[16,32]$.

Baicalein, evodiamine and luteolin are all classified as dual inhibitors of topoisomerase; but the latter compound did not promote any effect on T. cruzi cell proliferation. However, luteolin inhibits the growth of several cancer cell lines, blocking the cell cycle in the G1 phase [33]. In Leishmania, this inhibitor was also able to induce cell cycle arrest and apoptosis [18]. In this work, evodiamine promoted a slight inhibition of $T$. cruzi proliferation $\left(\mathrm{IC}_{50}\right.$ $90 \mu \mathrm{M})$ when compared to baicalein $\left(\mathrm{IC}_{50} 62.83\right)$, however this compound has presented efficacy against different cancer cell lines [20]. Dual inhibitors target topoisomerases I and II, thus it was expected that such compounds could present high efficiency in blocking cell proliferation and also promoting ultrastructural changes in the nucleus and kinetoplast; however these effects were not observed in T. cruzi after treatment with these inhibitors.

\section{Conclusions}

DNA topoisomerases represent an interesting target for anti-parasitic chemotherapy, since their inhibition interferes with the replicative process, which can lead to parasite death. In this work, we showed that compounds pertaining to the same topoisomerase inhibitor class had different effects on T. cruzi proliferation and ultrastructure. All inhibitors evaluated in this work are efficient for cancer therapy and sometimes blocked trypanosomatid growth, however their effects on $T$. cruzi proliferation and ultrastructure had never been investigated. Thus, we considered that they could be promissory agents in chemotherapeutic studies against $T$. cruzi, however these compounds presented considerably high $\mathrm{IC}_{50}$ values. The low effects observed in this parasite can be related

Table 1 Effects of topoisomerase I and dual inhibitors on Trypanosoma cruzi after $\mathbf{7 2} \mathbf{~ h}$ of treatment

\begin{tabular}{lccc}
\hline Drugs & Target & $\mathbf{I C}_{\mathbf{5 0}}(\boldsymbol{\mu M})$ & Ultrastructural effects \\
\hline Topotecan & Eukaryotic Topo I Inhibitor & 110 & Unpacking of nuclear heterochromatin and mitochondrial swelling \\
Irinotecan & Eukaryotic Topo I Inhibitor & $>300$ & No modifications \\
Baicalein & Eukaryotic Dual Inhibitor & 62,83 & No modifications \\
Luteolin & Eukaryotic Dual Inhibitor & $>300$ & No modifications \\
Evodiamine & Eukaryotic Dual Inhibitor & 90,73 & No modifications \\
\hline
\end{tabular}


to distinct factors such as the differences in human and protozoan topoisomerase domains, affinity for the target enzyme, cell membrane permeability and cell resistance, including mechanisms of drug efflux. Our results reinforce the idea that it is necessary to develop new compounds that may be successfully used in the therapy against neglected diseases.

\section{Additional files}

\section{Additional file 1: Cell viability of $T$. cruzi Y with irinotecan. T. cruzi} viability was not affected after treatment with irinotecan. The number of treated cells were similar to control parasites.

Additional file 2: Cell viability of $T$. cruzi Y with luteolin. T. cruzi viability was not affected after treatment with luteolin.

Additional file 3: The effects of dual inhibitors on the ultrastructure of $T$. cruzi epimastigotes. (A) T. cruzi treated with $50 \mu \mathrm{M}$ of baicalein for 72 h. (B) T. cruzi treated with $300 \mu \mathrm{M}$ of luteolin for 72 h. (C) T. cruzi treated with $300 \mu \mathrm{M}$ of evodiamine for $72 \mathrm{~h}$. Note that the nucleus and the kinetoplast preserved their typical organization. Bars $=1 \mu \mathrm{m}$. K, kinetoplast; ht, heterochromatin; nu, nucleolus; m, mitochondrion; f, flagellum.

\section{Competing interests}

The authors declare that they have no competing interests.

\section{Authors' contribution}

OL carried out the proliferation inhibition, ultrastructural and cell viability assays. AZ participated in the proliferation inhibition, ultrastructural and cell viability experiments and drafted the manuscript. CS carried out the ultrastructural and viability assays. WS conceived of the study and participated in the design of the manuscript. MCMM conceived of the study, designed and drafted the manuscript. All authors read and approved the manuscript.

\section{Acknowledgments}

We would like to thank to Rachel Rachid for technical assistance. This work was supported by Fundação Carlos Chagas Filho de Amparo à Pesquisa do Estado do Rio de Janeiro (FAPERJ) and Conselho Nacional de Desenvolvimento Científico e Tecnológico (CNPq).

\section{Author details}

'Laboratório de Ultraestrutura Celular Hertha Meyer, Instituto de Biofísica Carlos Chagas Filho, Universidade Federal do Rio de Janeiro, 21491-590 Rio de Janeiro, RJ, Brazil. "Instituto Nacional de Ciência e Tecnologia em Biologia Estrutural e Bioimagens, Universidade Federal do Rio de Janeiro, Rio de Janeiro, RJ, Brazil. ${ }^{3}$ Instituto Nacional de Metrologia, Qualidade e

Tecnologia-Inmetro, 20261-232 Duque de Caxias, RJ, Brazil.

Received: 10 January 2014 Accepted: 22 May 2014

Published: 10 June 2014

\section{References}

1. Rassi JRA, Rassi A, Resende MJ: American Trypanosomiasis (Chagas Disease). Infect Dis Clin North Am 2012, 26:275-291.

2. Ogbadoyi E, Ersfeld K, Robinson D, Sherwin T, Gull K: Architecture of the Trypanosoma brucei nucleus during interphase and mitosis. Chromosoma 2000, 108:501-513.

3. Elias MCQB, Faria M, Mortara RA, Motta MCM, De Souza W, Thiry M, Schenkman S: Chromosome localization changes in the Trypanosoma cruzi nucleus. Eukaryot Cell 2002, 1:944-953.

4. De Souza W: Basic cell biology of Trypanosoma cruzi. Curr Pharm Des 2002, 8:269-285.

5. Motta MCM, De Souza W, Thiry M: Immunocytochemical detection of DNA and RNA in endosymbiont-bearing trypanosomatids. FEMS Microbiol Letters 2013, 221:17-23.

6. Jensen RE, Englund PT: Network News: The replication of kinetoplast DNA. Annu Rev Microbiol 2012, 66:473-491.
7. Cortes F, Pastor N, Mateos S, Dominguez I: Roles of DNA topoisomerases in chromosome segregation and mitosis. Mutat Res 2013, 543:59-66.

8. Obado SO, Bot C, Nilsson D, Andersson B, Kelly JM: Repetitive DNA is associated with centromeric domains in Trypanosoma brucei but not Trypanosoma cruzi. Genome Biol 2007, 8:R37.

9. Wang JC: Cellular roles of DNA topoisomerases: a molecular perspective. Nat Rev Mol Cell Biol 2012, 3:430-440.

10. Champoux JJ: DNA Topoisomerases: structure, function, and mechanism. Annu Rev Biochem 2001, 70:369-413.

11. Hsiang YH, Lihou MG, Liu LF: Arrest of replication forks by drug-stabilized topoisomerase I-DNA cleavable complexes as a mechanism of cell killing by camptothecin. Cancer Res 1989, 49:5077-5082.

12. D'Arpa P, Beardmore C, Liu LF: Involvement of nucleic acid synthesis in cell killing mechanisms of topoisomerase poisons. Cancer Res 1990, 50:6919-6924.

13. Tsao YP, D'Arpa P, Liu LF: The involvement of active DNA synthesis in camptothecin-induced G2 arrest: altered regulation of $\mathrm{p} 34 \mathrm{cdc} / \mathrm{cyclin} \mathrm{B}$. Cancer Res 1992, 52:1823-1829.

14. Teicher BA: Next generation topoisomerase I inhibitors: rationale and biomarker strategies. Biochem Pharmacol 2008, 75:1626-1271.

15. Motta MCM: Kinetoplast as a potential chemotherapy target of trypanosomatids. Curr Pharm Des 2008, 14:847-854.

16. Li-Weber M: New therapeutic aspects of flavones: The anticancer properties of Scutellaria and its main active constituents Wogonin, Baicalein and Baicalin. Cancer Treat Rev 2009, 35:57-68.

17. Havsteen B: Flavonoids, a class of natural products of high pharmacological potency. Biochem Parmacol 1983, 32:1141-1148.

18. Mittra B, Saha A, Chowdhury AR, Pal C, Mandal S, Ukhopadahyay S, Bandyopadahyay S, Majumder HK: Luteolin, an abundant dietary component is a potent anti-leishmanial agent that acts by inducing topoisomerase II-mediated kinetoplast DNA Cleavage Leading to Apoptosis. Mol Med 2000, 6:527-541.

19. Das A, Dasgupta A, Sengupta T, Majumder HK: Topoisomerases of kinetoplastid parasites as potential chemotherapeutic targets. Trends Parasitol 2004, 20:381-386.

20. Chan ALF, Chang WS, Chen LM, Lee CM, Chen CE, Lin CM, Hwang JL: Evodiamine stabilizes topoisomerase I-DNA cleavable complex to inhibit topoisomerase I activity. Molecules 2009, 14:1342-1352.

21. Pan X, Hartley JM, Hartley JA, White KN, Wang Z, Bligh SWA: Evodiamine, a dual catalytic inhibitor of type I and II topoisomerases, exhibits enhanced inhibition against camptothecin resistant cells. Phytomedicine 2012, 7:618-624.

22. Camargo EP: Growth and differentiation in Trypanosoma cruzi. I. Origin of metacyclic trypanosomes in liquid media. Rev Inst Med Trop 1964, 6:93-100.

23. Henriques $C$, Moreira TLB, Maia-Brigagão C, Henriques-Pons A, Carvalho TMU, De Souza W: Tetrazoluim salt based methods for high-throughput evaluation of anti-parasite chemotherapy. Analytical Methods 2011, 3:2148-2155.

24. Pommier Y: Topoisomerase I, inhibitors: camptothecins and beyond. Nat Rev Cancer 2006, 6:789-802

25. Zuma AA, Cavalcanti DP, Maia MC, De Souza W, Motta MCM: Effect of topoisomerase inhibitors and DNA-binding drugs on the cell proliferation and ultrastructure of Trypanosoma cruzi. Int I Antimicrob Agents 2011, 37:449-456.

26. Cohen DP, Adams DJ, Flowers JL, Wall ME, Wani MC, Manikumar G, Colvin $\mathrm{OM}$, Silber R: Pre-clinical evaluation of $\mathrm{SN}-38$ and novel camptothecin analogs against human chronic B-cell lymphocytic leukemia lymphocytes. Leuk Res 1999, 23:1061-1070.

27. Bailly C: Homocamptothecins: potent topoisomerase I inhibitors and promising anticancer drugs. Crit Rev Oncol Hematol 2002, 45:91-108.

28. Deterding A, Dungey FA, Thompson K, Steverding D: Anti-trypanosomal activities of DNA topoisomerase inhibitors. Acta Tropica 2005, 93:311-316.

29. Prada CF, Alvarez-Velilla R, Balaña-Fouce R, Prieto C, Calvo-Alvarez E, Escudero-Martinez JM, Requena JM, Ordonez C, Desideri A, Perez-Pertejo Y, Reguera RM: Gimatecan and other camptothecin derivatives poison Leishmania DNA-topoisomerase IB leading to a strong leishmanicidal effect. Biochem Pharmacol 2013, 10:1433-1440.

30. Bodley AL, Shapiro TA: Molecular and cytotoxic effects of camptothecin, a topoisomerase I inhibitor, on trypanosomes and Leishmania. Proc Nat Acad Sci U S A 1995, 9:3726-3730. 
31. BoseDasgupta S, Das BB, Sengupta S, Ganguly A, Roy A, Dey S, Tripathi G, Dinda B, Majumder HK: The caspase-independent algorithm of programmed cell death in Leishmania induced bybaicalein: the role of LdEndoG, LdFEN-1 and LdTatD as a DNA 'degradesome'. Cell Death Differ 2008, 10:1629-1640.

32. Liu JJ, Huang TS, Cheng WF, Lu FJ: Baicalein and baicalin are potent inhibitors of angiogenesis: inhibition of endothelial cell proliferation, migration and differentiation. Int J Cancer 2003, 106:559-565.

33. Lin Y, Shi R, Wang X, Shen H: Luteolin, a flavonoid with potentials for cancer prevention and therapy. Curr Cancer Drug Targets 2008, 7:634-646.

doi:10.1186/1477-5751-13-11

Cite this article as: Lacombe et al:: Effects of camptothecin derivatives and topoisomerase dual inhibitors on Trypanosoma cruzi growth and ultrastructure. Journal of Negative Results in BioMedicine 2014 13:11.

\section{Submit your next manuscript to BioMed Central and take full advantage of:}

- Convenient online submission

- Thorough peer review

- No space constraints or color figure charges

- Immediate publication on acceptance

- Inclusion in PubMed, CAS, Scopus and Google Scholar

- Research which is freely available for redistribution 